\title{
Combined Treatment with Oral Kanamycin and Parenteral Antibiotics for a Case of Persistent Bacteremia and Intestinal Carriage with Campylobacter coli
}

\author{
Haruka Okada ${ }^{1}$, Takatoshi Kitazawa ${ }^{1}$, Sohei Harada ${ }^{1}$, Satoru Itoyama ${ }^{1}$, Shuji Hatakeyama ${ }^{1}$, \\ Yasuo Ota ${ }^{1,2}$ and Kazuhiko Koike ${ }^{1}$
}

\begin{abstract}
Campylobacter coli (C. coli) is a rare pathogen of bacteremia, but in immunocompromised hosts, C. coli occasionally causes bacteremia which can be refractory to antibiotic treatment. We report a case of $C$. coli bacteremia in a patient with X-linked agammaglobulinemia. Bacteremia relapsed repeatedly in spite of treatment with combined intravenous antibiotics. $C$. coli was observed in the biopsy specimens from the intestinal mucosa, suggesting intestinal carriage and reservoir of recurring infection. The addition of oral kamamycin with intravenous antibiotics was successful in eradicating $C$. coli from the blood and intestine.
\end{abstract}

Key words: Campylobacter coli, X-linked agammaglobulinemia, oral kamamycin

(Inter Med 47: 1363-1366, 2008)

(DOI: 10.2169/internalmedicine.47.1161)

\section{Introduction}

Campylobacter jejuni and Campylobacter coli (C. coli) frequently cause enteritis, but rarely cause bacteremia or extraintestinal infections in immunologically normal hosts $(1,2)$. However, in immunocompromised hosts, especially in patients with humoral immunodeficiency, these organisms occasionally develop prolonged, severe extraintestinal infection such as bacteremia, osteomyelitis, and arthritis (1, 3-5). We report a case of bacteremia, endocarditis, and osteomyelitis with $C$. coli in a patient with $\mathrm{X}$-linked agammaglobulinemia. Bacteremia relapsed repeatedly in spite of treatment with combined intravenous antibiotics. Campylobacter was never isolated from stool cultures, but observed in the biopsy specimens from intestinal mucosa, suggesting the intestinal carriage. The addition of oral kamamycin with intravenous antibiotics was successful in eradicating $C$. coli from the blood, the bone and the intestine.

\section{Case Report}

A 33-year-old man with X-linked agammaglobulinemia was admitted to our hospital because of sustained fever and cellulitis of the left leg. The patient had history of recurrent diarrhea, upper respiratory infection and otitis media, and had been receiving immunoglobulin replacement therapy every two weeks. He had no history of overseas travel. He did not remember having raw meat such as chicken or coming into contact with animals in the past year. At the outpatient visits, oral cefcapene pivoxil and then intravenous ceftriaxone were administered, but the fever and cellulitis did not improve.

On admission, his temperature was $39.5^{\circ} \mathrm{C}$, and his left leg was swollen. A grade 1/6 holosystolic cardiac murmur was heard. The abdomen was normal. The white blood cell count was $2,700 / \mathrm{mm}^{3}$, the $\mathrm{C}$ reactive protein level was $1.09 \mathrm{mg} / \mathrm{dL}$. The serum IgG was $893 \mathrm{mg} / \mathrm{dL}$, but neither IgA nor IgM was detectable. Meropenem and then intravenous ciprofloxacin were administered for ten days. Seven days after discontinuation of the intravenous antibiotics, cel-

${ }^{1}$ Department of Infectious Diseases, Graduate School of Medicine, The University of Tokyo, Tokyo and ${ }^{2}$ Department of Medicine, Teikyo University School of Medicine, Tokyo

Received for publication March 26, 2008; Accepted for publication April 25, 2008

Correspondence to Dr. Yasuo Ota, yasuota-tky@umin.ac.jp 


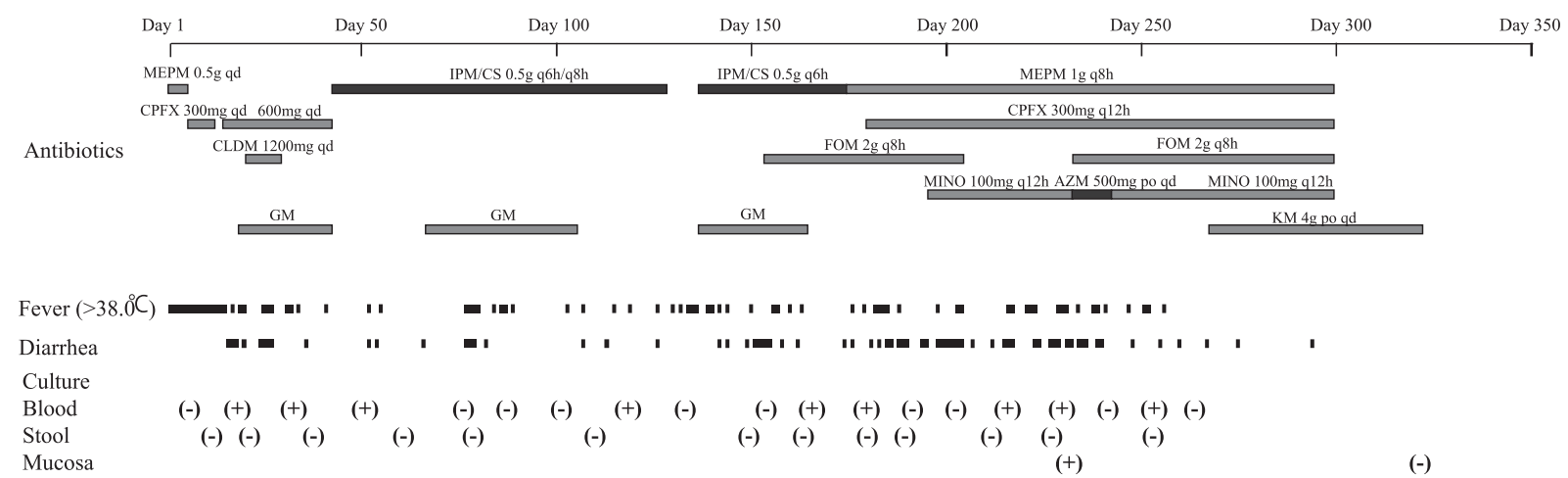

Figure 1. Clinical course of the patient. Antibiotics are shown in the upper panel. Fever and diarrhea are shown in the middle panel. Cultures from blood, stool and the intestinal mucosa are shown in the lower panel. MEPM: meropenem, IPM/CS: imipenem/cilastatin sodium, CPFX: ciprofloxacin, CLDM: clindamycin, FOM: fosfomycin, MINO, minocycline, AZM: azithromycin, GM: gentamicin, KM: kanamycin.

lulitis of his left leg worsened again. Blood culture was positive for Campylobacter species, which was determined as $C$. coli by a polymerase chain reaction assay. Stool cultures obtained repeatedly during the hospitalization did not yield C. coli (Fig. 1).

Intravenous administration of ciprofloxacin and gentamicin was started. Blood cultures continued to be positive for C. coli. A transesophageal echocardiography showed vegetation on the mitral valve, which suggested infective endocarditis. A colon fiberscope revealed mild inflammation. Antibiotics were switched to imipenem/cilastatin, and one week after the switch, blood culture turned negative. But four days after discontinuation of the 8-week antibiotic therapy, he became febrile again, and blood culture turned positive for C. coli. Intravenous imipenem/cilastatin and gentamicin were restarted. A magnetic resonance imaging study of the lower extremities revealed osteomyelitis of the left leg. Fosfomycin, which was susceptible in MIC test, was added to imipenem/cilastatin and gentamicin (Table 1). Gentamicin was discontinued because of renal tubular injury. Failing to eradicate $C$. coli from the blood, imipenem/cilastatin was switched to meropenem, ciprofloxacin and minocycline according to the susceptibility tests, and oral azithromycin, were sequentially added. In spite of disappearance of vegetation on the mitral valve in a transesophageal echocardiography and resolution of osteomyelitis of the left leg, bacteremia with $C$. coli continued (Fig. 1). During the combined antibiotic therapy, diarrhea and abdominal fullness became more prominent, although toxin A of Clostridium difficile was not detected from the stool. A colon fiberscope was performed again. The ascending colon was edematous, and the terminal ileum and the hepatic flexure were erosive. Although bacteria could not be detected histologically, all cultures of biopsy specimens sampled from the mucosa of the colon and the ileum yielded Campylobacter spp., suggesting intestinal carriage and reservoir of recurring infection.

Oral kanamycin was added to the intravenous antibiotics (Fig. 1). The following day his fever was resolved, and the
Table 1. The Minimum Inhibitory Concentrations to Antimicrobial Agents against Campylobacter Coli Isolated from the Blood of Our Patient

\begin{tabular}{lr}
\hline Antimicrobial agents & $\begin{array}{r}\text { MIC } \\
(\mu \text { g/mL })\end{array}$ \\
\hline Ampicillin & 16.0 \\
Sulbactam/ampicillin & 32.0 \\
Cefotaxime & 128.0 \\
Cefpirome & $>32.0$ \\
Aztreonam & $>64.0$ \\
Imipenem/cilastatin & $<0.5$ \\
Meropenem & $<0.5$ \\
Gentamicin & $<4.0$ \\
Erythromycin & 8.0 \\
Clarythromycin & 8.0 \\
Minocycline & 16.0 \\
Ciprofloxacin & 8.0 \\
Fosfomycin & $<8.0$ \\
\hline
\end{tabular}

MIC: minimum inhibitory concentration

abdominal symptoms improved. Blood cultures remained negative and intravenous antibiotics were discontinued six weeks after blood culture turned negative. A colon fiberscope was performed again, and oral kanamycin was discontinued after negativity of cultures of biopsy specimens from intestinal mucosa was confirmed. The total duration of oral kanamycin was ten weeks. The patient has been well for about eight months without relapse of bacteremia and diarrhea.

\section{Discussion}

Campylobacter coli is one of the most common Campylobacter species associated with diarrhea illness other than $C$. 
jejuni, and these two species produce clinically indistinguishable infections (6). C. coli are present not only in food animals such as poultry, cattle, sheep, pigs, but also in domestic pets. Since Campylobacter species other than C. jejuni are difficult to identify with phenotypic testing, tests for detection of species-specific sequences via PCR have been developed (7). Bacteremia caused by Campylobacter species is uncommon and usually resolves spontaneously in immunologically normal hosts (2). In compromised hosts however, prolonged, severe and recurrent Campylobacter bacteremia and other extraintestinal infections may occur (2-4). In such patients, intestinal tissue invasion of Campylobacter spp. may be one of the pathogenetic mechanisms $(2,5)$. In normal subjects with $C$. jejuni/coli enterocolitis, serum IgA, $\mathrm{IgM}$, and $\mathrm{IgG}$ antibodies to $C$. jejuni/coli rise rapidly after infection, and IgA antibodies in intestinal secretions also increase $(8,9)$. In patients with hypogammaglobulinemia or infected with human immunodeficiency virus (HIV), an impaired antibody response to $C$. jejuni/coli infection including bacteremia has been noted $(10,11)$.

There have been eleven case reports of X-linked agammaglobulinemia with Campylobacter bacteremia (12-19). Among them six cases had stool cultures positive for Campylobacter species and the other five cases had no description about stool cultures. Furthermore, among the six positive stool culture cases, five cases had no gastrointestinal symptoms such as abdominal pain or diarrhea. We speculated that intestinal carriage of Campylobacter with or without gastrointestinal symptoms might be a risk factor of recurrent bacteremia in immunocompromised patients. Stool cultures had sensitivity of $40 \%$ for Campylobacter bacteremia (1). In the present patient, stool cultures were consistently negative for $C$. coli, but cultures of biopsy specimens from intestinal mucosa were positive for $C$. coli. Furthermore in our case, infective endocarditis and osteomyelitis were clinically resolved in spite of persistent bacteremia. We speculated that the intestinal tract would be a reservoir for C. coli. The mechanism of intestinal carriage of Campylobacter in immunocompromised patients remained unclarified, but it has been supposed that failure of humoral immune response in these patients might permit colonization of Campylobacter in the epithelium and lamina propria and induction of tissue damage $(20,21)$. A study on Campylo- bacter infections in 38 HIV-infected patients reported that in two patients with diarrhea, the cultures were positive only in the blood, and that in one patient a culture of biopsy specimen from intestinal mucosa yielded $C$. jejuni after disappearance in stool culture (22). We consider that in compromised patients, who are suspected to be infected with Campylobacter but have negative stool cultures for bacteria, culture of intestinal mucosa might be useful for diagnosing the intestinal carriage.

Macrolides and fluoroquinolones are antibiotic agents frequently used to treat Campylobacter infection (2). However, in $C$. coli bacteremia in compromised patients, treatment with these drugs might fail, and combined therapy of intravenous antibiotics such as carbapenems and aminoglycosides has been used (18). There have been three reports in which administration of oral antibiotics in addition to intravenous antibiotics were effective in eradicating $C$. coli from the intestine $(12,15,18)$. In the present case, oral antibiotics other than azithromycin had not been tried until isolation of $C$. coli from the culture of intestinal mucosa, because the intestinal carriage had not been considered from mild nonspecific inflammatory findings in the first colonoscope and repeated negative stool culture. Intestinal biopsy was not performed in the previous reports, and this is the first case in which culture of intestinal mucosa was used for diagnosing the intestinal carriage with C.coli and confirming the eradication by the treatment with oral antibiotics. Oral kanamycin was selected in our case because the isolated Campylobacter strain was susceptible to gentamicin. Since aminoglycosides including kanamycin have poor bioavailability, we considered that oral administration of kanamycin could not reach a sufficiently high serum concentration to treat endocarditis or osteomyelitis, but the administration could reach a sufficient concentration in the intestinal mucosa for eradicating Campylobacter spp. from the intestine. We suggested that in a case of bacteremia and intestinal carriage with Campylobacter which was refractory to intravenous antibiotics, oral aminoglycoside therapy combined with intravenous antibiotics could be effective.

\section{Acknowledgement}

We would like to thank Mie Kataoka for preparing the manuscript.

\section{References}

1. Pigrau C, Bartolome R, Almirante B, Planes AM, Gavalda J, Pahissa A. Bacteremia due to Campylobacter species, clinical findings and antimicrobial susceptibility patterns. Clin Infect Dis 25: 1414-1420, 1997.

2. Blaster MG, Bennett JE, Dolin R. Campylobacter jejuni and related species. In: Mandell, Douglas and Bennette's Principles and Practice of Infectious Diseases. 6th ed. Mandell GL, Douglas RG, Bennett JE, Eds. Elsevier/Churchill Livingstone, New York, 2005: 2276-2285.

3. Peterson MC. Clinical aspects of Campylobacter jejuni infections in adults. West J Med 161: 148-152, 1994.
4. Akiba T, Akiba K, Suto N, Kumagai K, Sakamoto M, Yazaki N. Campylobacter coli bacteremia in an 11-year-old boy. Pediatr Int 44: 543-544, 2002.

5. Ladron de, Guevara C, Gonzalez J, Pena P. Bacteraemia caused by Campylobacter spp. J Clin Pathol 47: 174-175, 1994.

6. Fitzgerald C, Dolin R. Campylobacter and Arcobacter. In: Manual of Clinical Microbiology. 9th ed. Murray PR, Baron EJ, Jorgensen $\mathrm{JH}$, et al, Eds. American Society for Microbiology, Washington, DC, 2007: 933-946.

7. Gonzalez I, Grant KA, Richardson PT, Park SF, Collins MD. Specific identification of the enteropathogens Campylobacter jejuni 
and Campylobacter coli by using a PCR test based on the ceuE gene encoding a putative virulence determinant. J Clin Microbiol 35: 759-763, 1997.

8. Black RE, Perlman D, Clements ML. Human volunteer studies with Campylobacter jejuni. In: Campylobacter jejuni. Current Status and Future Trends. Nachmkin I, Blaser MJ, Topmpkins LS, Eds. American Society for Microbiology, Washington DC, 1992: 207-215.

9. Black RE, Levine MM, Clements ML, Hughes TP, Blaser MJ. Experimental Campylobacter jejuni infection in humans. J Infect Dis 157: 472-479, 1988.

10. Johnson RJ, Nolan C, Wang SP, Shelton WR, Blaser MJ. Persistent Campylobacter jejuni infection in an immunocompromised patient. Ann Intern Med 100: 832-834, 1984.

11. Perlman DM, Ampel NM, Schifman RB, et al. Persistent Campylobacter jejuni infections in patients infected with the human immunodeficiency virus (HIV). Ann Intern Med 1108: 540-546, 1988.

12. Arai A, Kitano A, Sawabe E, Kanegane H, Miyawaki T, Miura O Relapsing Campylobacter coli bacteremia with reactive arthritis in a patient with X-linked agammaglobulinemia. Intern Med 46: 605609, 2007.

13. van der Meer JW, Mouton RP, Daha MR, Schuurman RK. Campylobacter jejuni bacteraemia as a cause of recurrent fever in a patient with hypogammaglobulinaemia. J Infect 12: 235-239, 1986.

14. Spelman DW, Davidson N, Buckmaster ND, Spicer WJ, Ryan P.
Campylobacter bacteraemia: a report of 10 cases. Med J Aust 145: 503-505, 1986

15. Chusid MJ, Coleman CM, Dunne WM. Chronic asymptomatic Campylobacter bacteremia in a boy with X-linked hypogammaglobulinemia. Pediatr Infect Dis J 6: 943-944, 1987.

16. Autenrieth IB, Schuster V, Ewald J, Harmsen D, Kreth HW. An unusual case of refractory Campylobacter jejuni infection in a patient with X-linked agammaglobulinemia: successful combined therapy with maternal plasma and ciprofloxacin. Clin Infect Dis 23: 526-531, 1996.

17. Rafi A, Matz J. An unusual case of Campylobacter jejuni pericarditis in a patient with X-linked agammaglobulinemia. Ann Allergy Asthma Immunol 89: 362-367, 2002.

18. Tokuda K, Nishi J, Miyanohara H, et al. Relapsing cellulitis associated with Campylobacter coli bacteremia in an agammaglobulinemic patient. Pediatr Infect Dis J 23: 577-579, 2004.

19. Van der Hilst JC, Smits BW, van der Meer JW. Hypogammaglobulinaemia: cumulative experience in 49 patients in a tertiary care institution. Neth J Med 60: 140-147, 2002.

20. Freeman AF, Holland SM. Persistent bacterial infections and primary immune disorders. Curr Opin Microbiol 10: 70-75, 2007.

21. Wooldridge KG, Ketley JM. Campylobacter-host cell interactions. Trends Microbiol 5: 96-102, 1997.

22. Molina J, Casin I, Hausfater P, et al. Campylobacter infections in HIV-infected patients: clinical and bacteriological features. Aids 9: 881-885, 1995.

(C) 2008 The Japanese Society of Internal Medicine http://www.naika.or.jp/imindex.html 